\title{
SOME HILBERT SPACE EXTREMAL PROBLEMS
}

\author{
MARVIN ROSENBLUM ${ }^{1}$
}

In this note we shall generalize in several ways the following theorem of Philip Davis:

Let $l^{2}$ be a real or complex sequential Hilbert space. Fix $\left\{h_{j}\right\}_{0}^{\infty}$ in $l^{2}$, a positive real number $M$, and a sequence $\left\{k_{j}\right\}_{0}^{\infty}$ such that $k_{j}>0$ for all $j$ and $\sum_{j=0}^{\infty} k_{j}^{-1}$ is finite. Suppose that $\sum_{j=0}^{\infty} k_{j}\left|h_{j}\right|^{2}>M^{2}$.

Then there exists a unique element $\left\{e_{j}\right\} \in l^{2}$ such that the infimum in

$$
\inf \left\{\sum_{j=0}^{\infty}\left|h_{j}-a_{j}\right|^{2}:\left\{a_{j}\right\} \in l^{2}, \sum_{j=0}^{\infty} k_{j}\left|a_{j}\right|^{2} \leqq M^{2}\right\}
$$

is achieved. This is given by $e_{j}=\left(1+\lambda k_{j}\right)^{-1} h_{j}$, where $\lambda$ is the unique positive root of $\sum_{j=0}^{\infty} k_{j}\left(1+\lambda k_{j}\right)^{-1}\left|h_{j}\right|^{2}=M^{2}$.

Davis proved the result by first considering the extremal problem for $n$ dimensional Euclidean space $R^{n}$. In this case the problem has the following geometric interpretation:

$\left(\alpha^{\prime \prime}\right)$ Let $\mathrm{E}$ be the hyperellipse $\left\{\left(a_{1}, \cdots, a_{n}\right): \sum_{j=0}^{n} k_{j} a_{j}^{2} \leqq M^{2}\right\}$ in $R^{n}$ and suppose that $h=\left(h_{1}, \cdots, h_{n}\right)$ is not in $\mathrm{E}$. Find the vector $e$ in E such that the distance from $h$ to $e$ is a minimum.

This $R^{n}$ problem is readily handled by Lagrange multipliers. The growth condition on the $k_{j}$ permits the use of finite dimensional approximations to deduce the more general result. See $[1]$ or $[2$, p. 204].

Our first generalization of Davis' theorem is

THEOREM 1. Let $\mathfrak{H C}$ be a real or complex Hilbert space, and suppose $h \in \mathcal{F}$. Suppose $A$ is a closed linear operator on $\mathfrak{H C}$ to $\mathfrak{H C}$ whose domain $D(A)$ is dense in FC. Fix $M>0$.

Then there is a unique element $e$ in $3 C$ such that the infimum in

$$
\inf \{\|h-a\|:\|A a\| \leqq M\}
$$

is achieved.e $=h$ if and only if $\|A h\| \leqq M$. If $\neq \neq h$, then $e=\left(I+\lambda A^{*} A\right)^{-1} h$, where $\lambda$ is the unique positive solution of $\left\|A\left(I+\lambda A^{*} A\right)^{-1} h\right\|=M$.

It is clear that $\left(\alpha^{\prime}\right)$ is a special case of $(\alpha)$. If $A$ is specialized so that $A^{-1}$ exists and is a Schmidt-Hilbert operator, then Davis' theorem and Theorem 1 are in fact equivalent.

Our proof of Theorem 1 is carved up into three lemmas. Through-

Received by the editors May 11, 1964.

1 This research was partially supported by National Science Foundation Grant 18853. 
out we assume that $\mathfrak{H}, h, A$ and $M$ are as stated in the hypotheses of Theorem 1.

Lemma 1. There exists a unique extremal element e minimizing $(\alpha)$.

Proof. $\{a:\|A a\| \leqq M\}$ is clearly a convex set, we will show that it is closed and this will imply Lemma 1 . Suppose $a_{n} \rightarrow a$ and $\left\|A a_{n}\right\| \leqq M$. Extract a subsequence $\left\{b_{n}\right\}$ of $\left\{a_{n}\right\}$ such that $A b_{n}$ converges weakly, say, to $b$. Thus for any $c \in \mathbb{D}\left(A^{*}\right)$

$$
\langle b, c\rangle=\lim _{n \rightarrow \infty}\left\langle A b_{n}, c\right\rangle=\lim _{n \rightarrow \infty}\left\langle b_{n}, A^{*} c\right\rangle=\left\langle a, A^{*} c\right\rangle .
$$

Thus $A^{* *} a=b$, and since $A$ is closed, $A a=b .\|A a\| \leqq \lim \inf _{n \rightarrow \infty}\left\|A a_{n}\right\|$ $\leqq M$, so $a$ is in the convex set.

The following lemma is basic for our proof, and despite appearances, is quite geometrical in nature. Suppose $\mathfrak{H}=R^{n}$ and consider the situation of $\left(\alpha^{\prime \prime}\right) .2$ (i) states that if $a$ is tangent to the boundary of $\mathrm{E}$ at $e$, then $h-e$ is perpendicular to $a$. 2(ii) shows that the angle between $h-e$ and $e$ satisfies $-\pi / 2 \leqq \theta \leqq \pi / 2$.

Lemma 2. (i) If $a \in \mathfrak{D}(A)$ and $\langle A e, A a\rangle=0$, then $\langle h-e, a\rangle=0$. In particular, if $A e=0$ then $e=h$.

(ii) $\langle h-e, e\rangle \geqq 0$.

Proof. We shall give the proof for complex Hilbert spaces only as the real case can be then handled by simple modifications. Assume the hypotheses of (i). With no loss in generality we may assume that $\|A a\| \leqq M$. Let $\mu$ and $\nu$ be complex numbers such that $|\mu|^{2}+|\nu|^{2} \leqq 1$. Then $\|A(\mu e+\nu a)\| \leqq M$. Thus $0 \leqq\|h-\mu e-\nu a\|^{2}-\|h-e\|^{2}$, so 0 $\leqq 2 \operatorname{Re}\left[\left(1-\mu^{*}\right)\langle h-e, e\rangle\right]-2 \operatorname{Re}\left[\nu^{*}\langle h-e, a\rangle\right]+\|(1-\mu) e-\nu a\|^{2}$, where * is the complex conjugation operation. We derive (i) and (ii) by selecting suitable $\mu$ and $\nu$.

To prove (i) choose $\mu=1-t, 0<t<1$, and $\nu=\left(2 t-t^{2}\right)^{1 / 2} \operatorname{sgn}\langle h-e, a\rangle$. Then $0 \leqq-2\left(2 t-t^{2}\right)^{1 / 2}|\langle h-e, a\rangle|+O(t)$ as $t \rightarrow 0+$, and this implies (i).

Next we prove (ii). Pick $\theta$ so $\cos \theta>0$, and set $\mu^{*}=1-e^{i \theta} t, 0<t$, $t$ small, $a=0$, and $\nu=0$. Then $0 \leqq 2 t \operatorname{Re}\left[e^{i \theta}\langle h-e, e\rangle\right]+O\left(t^{2}\right)$ as $t \rightarrow 0+$, so $\operatorname{Re}\left[e^{i \theta}\langle h-e, e\rangle\right] \geqq 0$. This implies (ii).

LEMma 3. Either $e=h$ or there exists a unique positive number $\lambda$ such that $\left(I+\lambda A^{*} A\right) e=h$.

Proof. If $A e=0$ then Lemma 2(i) guarantees that $e=h$. Thus we may assume that $A e \neq 0$. Let $b$ be in $D\left(A^{*} A\right) \subset D(A)$ and consider $a=\|A e\|^{2} b-\langle A b, A e\rangle e$. Then $\langle A e, A a\rangle=0$, so we may invoke Lemma 2(i) to deduce that $\langle h-e, a\rangle=0$. It follows that $\langle h-e, b\rangle=\lambda\langle A e, A b\rangle$, 
where $\lambda=\|A e\| \|^{-2}\langle h-e, e\rangle \geqq 0$. If $\lambda=0$ then $h-e$ is perpendicular to $D\left(A^{*} A\right)$, so $h=e$. Assume then that $\lambda>0$. We have $\langle h, b\rangle$ $=\left\langle e,\left(I+\lambda A^{*} A\right) b\right\rangle$ for all $b \in \mathbb{D}\left(A^{*} A\right)$, so $\left(I+\lambda A^{*} A\right) e=h$. The condition $A e \neq 0$ implies that $\lambda$ is unique.

Proof of Theorem 1. Suppose it is not the case that $\|A h\| \leqq M$, so $e \neq h$. Then there exists $\lambda>0$ such that

$$
\|h-e\|=\left\|h-\left(I+\lambda A^{*} A\right)^{-1} h\right\|=\left\|\lambda A^{*} A\left(I+\lambda A^{*} A\right)^{-1} h\right\| .
$$

This last expression is a strictly increasing function of $\lambda$. Now, $\|A e\|^{2}=\left\langle A^{*} A\left(I+\lambda A^{*} A\right)^{-2} h, h\right\rangle$. This is a strictly decreasing function of $\lambda$ that approaches a number greater than $M$ or diverges to infinity as $\lambda \rightarrow 0+$. Thus necessarily $\|A e\|=M$.

An alternate formulation of Davis' theorem considers the extremal problem

$$
\inf \left\{\sum_{j=0}^{\infty}\left|h_{j}-k_{j}^{-1 / 2} b_{j}\right|^{2}: \sum_{j=0}^{\infty}\left|b_{j}\right|^{2} \leqq M\right\} .
$$

This problem is equivalent to $\left(\alpha^{\prime}\right)$ by an obvious change of variables. We turn now to a generalization of $\left(\beta^{\prime}\right)$.

THEOREM 2. Let $3 \mathrm{C}$ be a complex Hilbert space, $h$ an element of $\mathcal{H C}$ and $M$ a positive real number. Suppose $B$ is a closed densely defined linear operator on $\mathfrak{H C}$ to $\mathfrak{H C}$ with polar decomposition $B=W|B|$, so $W W^{*}$ is the projection operator on the closure of the range of $B$.

Then there is a unique element Bf in $\mathfrak{H C}$ such that the infimum in

$$
\inf \{\|h-B b\|:\|b\| \leqq M\}=\beta(h)
$$

is achieved. $B f=W W^{*} h$ if and only if there exists $b$ with $\|b\| \leqq M$ and $W W^{*} h=B b$. If $B f \neq W W^{*} h$, then

$$
B f=W|B|^{2}\left(|B|^{2}+\lambda I\right)^{-1} W^{*} h,
$$

where $\lambda$ is the unique positive solution of

$$
\left\||B|\left(|B|^{2}+\lambda I\right)^{-1} W^{*} h\right\|=M \text {. }
$$

Let $B$ and $W$ be as in Theorem 2. First we prove

LEMma 4. There is a unique extremal element Bf minimizing $(\beta)$.

Proof. $\{B b:\|b\| \leqq M\}$ is a convex set. The same technique used in the proof of Lemma 1 shows that it is closed.

We fix $B f$ as the above extremal element. $|B|=\left(B^{*} B\right)^{1 / 2}$ is a self-adjoint operator such that $D(|B|)=\mathscr{D}(B)$ and $\|B b\|=\||B| b\|$ for 
all $b \in D(B) . B$ and $|B|$ have the same nullspace, $\left(I-W^{*} W\right)$ FC. (See [3, pp. 304-308] for a discussion of the polar decomposition.) In considering problem $(\beta)$ it suffices to consider $b$ orthogonal to this nullspace, that is, $b$ in $W^{*} W \mathcal{H}$. There is a self-adjoint operator $A$ such that $A|B| b=W^{*} W b$ for all $b$ in $D(B) ; A$ is the inverse of $|B|$ relative to the Hilbert space $W^{*} W \mathcal{H}$. $W^{*} W$ is the projection operator on the closure of the range of $|B|$.

LEMMA 5.

$$
\beta^{2}(h)=\|h\|^{2}-\left\|W^{*} h\right\|^{2}+\inf \left\{\left\|W^{*} h-a\right\|^{2}:\|A a\| \leqq M\right\} .
$$

This last infimum is uniquely achieved by an element $e$ in $D(A)$ $\subset W^{*} W$ FC. $B f=W e$.

Proof. If $b$ is in $D(A)$, then

$$
\begin{aligned}
\|h-B b\|^{2} & =\|h-W|B| b\|^{2} \\
& =\|h\|^{2}-\left\langle|B| b, W^{*} h\right\rangle-\left\langle W^{*} h,|B| b\right\rangle+\||B| b\|^{2} \\
& =\|h\|^{2}-\left\|W^{*} h\right\|^{2}+\left\|W^{*} h-|B| b\right\|^{2} .
\end{aligned}
$$

Thus

$$
\begin{aligned}
\beta^{2}(h)= & \|h\|^{2}-\left\|W^{*} h\right\|^{2} \\
& \left.+\inf \left\{\left\|W^{*} h-|B| b\right\|^{2}:\|b\| \leqq M, b \in W^{*} W \Im\right\}\right\} \\
= & \|h\|^{2}-\left\|W^{*} h\right\|^{2}+\inf \left\{\left\|W^{*} h-a\right\|^{2}:\|A a\| \leqq M\right\} .
\end{aligned}
$$

An application of Theorem 1 shows that this last extremal problem has a unique solution $e$. Necessarily $|B| f=e$, so $B f=W e$.

Proof of Theorem 2. We list a quadruple of equivalent statements: $B f=W W^{*} h ; \quad e=W^{*} h ;\left\|A W^{*} h\right\| \leqq M$; there exists $b$ with $\|b\| \leqq M$ and $W W^{*} h=B b$. The first two are equivalent since $W$ is a partial isometry, the next are equivalent by Theorem 1 as applied to the situation of Lemma 5. The final equivalence is a consequence of the definition of $A$.

Next suppose that $e \neq W^{*} h$. Then by applying Theorem 1 to the problem in Lemma 5 we see that the infimum in $(\beta)$ is achieved by $B f=W e=W\left(I+\lambda A^{2}\right)^{-1} W^{*} h=W|B|^{2}\left(|B|^{2}+\lambda I\right)^{-1} W^{*} h$, where $\lambda$ is the unique positive solution of

$$
M=\left\|A\left(I+\lambda A^{2}\right)^{-1} W^{*} h\right\|=\left\||B|\left(|B|^{2}+\lambda I\right)^{-1} W^{*} h\right\| .
$$

When applying Theorem 2 it is sometimes convenient to note that (2.1) and (2.2) can be simplified if $h$ is in $D\left(B^{*}\right)$. (2.1) becomes

$$
B f=B\left(B^{*} B+\lambda I\right)^{-1} B^{*} h
$$


and (2.2) becomes

$$
\left\|\left(B^{*} B+\lambda I\right)^{-1} B^{*} h\right\|=M .
$$

As an elementary application we have

Theorem 3. Let $D(D)$ be the set of all $b$ in the complex Lebesgue space $\mathrm{HC}=L^{2}(-\infty, \infty)$ such that $b$ is locally absolutely continuous on $(-\infty, \infty)$ and whose derivative $b^{\prime}$ is in $3 C . D$ sends any $b$ in $D(D)$ into $i b^{\prime}$. Let $h$ be in $L^{2}(-\infty, \infty)$ and $M$ be a positive real number.

Then the extremal problem inf $\{\|h-D b\|:\|b\| \leqq M\}$ has a unique extremal element $D f$. Either $h=D f$ or

$$
(D f)(x)=h(x)-\frac{\omega}{2} \int_{-\infty}^{\infty} h(t) \exp (-\omega|x-t|) d t
$$

for some real positive number $\omega$.

Proof. As shown in Stone [4, pp. 441-445], $D=F S F *$, where $\mathcal{F}$ is the Fourier-Plancherel transform and $\delta$ is the self-adjoint operator that sends $f(x)$ into $x f(x)$. Then by Theorem 2 either $h=D f$, or, by $(2.1)^{\prime}, D f=D^{2}\left(D^{2}+\omega^{2} I\right)^{-1} h=h-\omega^{2} \mathfrak{F}\left(\mathcal{S}^{2}+\omega^{2} I\right)^{-1} \mathfrak{F} * h$, where $\omega>0$. Thus (3.1) holds.

\section{REFERENCES}

1. Philip Davis, An application of doubly orthogonal functions to a problem of approximation in two regions, Trans. Amer. Math. Soc. 72 (1952), 104-137.

2. H. Meschkowski, Hilbertsche Räume mit Kernfunction, Springer, Berlin, 1962.

3. J. von Neumann, Über Adjungierte Funktionaloperatoren, Ann. of Math. 33 (1932), 294-310.

4. M. H. Stone, Linear transformations in Hilbert space and their applications to analysis, Amer. Math. Soc. Colloq. Publ. Vol. 15, Amer. Math. Soc., Providence, R. I., 1932.

UNIVERSITY OF VIRGINIA 